\title{
Environmental effects in galaxies - searching for massive stars
}

\author{
Duíla F. de Mello ${ }^{1}$, Marcio A.G. Maia ${ }^{2}$, Daniel Schaerer ${ }^{3}$, \\ and Claus Leitherer ${ }^{1}$ \\ ${ }^{1}$ Space Telescope Science Institute, Baltimore, MD, USA \\ ${ }^{2}$ Observatório Nacional, Rio de Janeiro, Brazil \\ ${ }^{3}$ Observatoire Midi-Pyrénées, Toulouse, France
}

\begin{abstract}
In order to study the influence of the environment in galaxies we have selected a sample from the catalog by Maia et al. (1994) which contains objects in low and high density areas of the Southern sky. These samples have been observed with the Brazilian-ESO 1.52m telescope and long-slit spectra are being analyzed. With our database we will be able to investigate the role of the environment in the galaxy population. We will be able to search for WR features and massive stars. It is our goal to compare the general properties of these galaxies with typical starburst galaxies which are dominated by episodes of massive star formation.
\end{abstract}

\section{Introduction}

It is well known that interaction plays a fundamental role in galaxy evolution. However, there are many issues concerning environmental effects that are still not well established. For instance, can interaction play a role in defining the stellar population of galaxies? Is the strength of the starburst related to the type of interaction? In this study we have started searching for massive stars in galaxies at different environments. The detection of massive stars in these galaxies will allow us to date the last burst of star formation precisely. This result will be used to search for a correlation between the environment where the galaxy lives, the type of interaction and the stellar population.

The sample we have chosen is that of Maia et al. (1994). The high density sample (HDS) is formed by galaxies that are in groups of three or more members and are in areas with densities larger than 18 galaxies/ $\mathrm{Mpc}^{3}$. The low density sample (CS) is formed by galaxies which are not members of any group and are in areas with densities less than 0.0004 galaxies/ $\mathrm{Mpc}^{3}$. The HDS and CS samples contain 151 and 179 galaxies, respectively.

We have started a systematic search for massive stars and Wolf-Rayet stars. Our motivation was based on the numerous serendipitous discoveries of WR galaxies in the literature (see Schaerer et al. 1999 for a catalog of WR galaxies).

A preliminary stellar population analysis showed that the frequency of the stellar population types of the HDS does not present significant differences when compared to the low local density environment sample (Pastoriza et al. 1994). Most of the HDS galaxies were found to host an AGN or to be in a transition between an HII region and AGN. The CS has only spectra typical of $\mathrm{H}$ II regions. 
This suggests that a high density environment could be inducive to gas transfer towards the nucleus, but not to enhance star formation activity. However, their sample had only 20 galaxies from the HDS and 18 from the CS sample.

Recently, more spectroscopic data were obtained as part of a key project for the Observatório Nacional (Brazil) $50 \%$ share of the ESO $1.52 \mathrm{~m}$ spectroscopic telescope on La Silla, Chile. A total of 100 galaxies have been observed, 50 belonging to the HDS and 50 to the CS. We have started a systematic search for massive stars and WR stars.

\section{Identifying massive stars}

We are searching for typical features of WR stars, such as the WR bump where the He II $\lambda 4686$ line is located. We have observed a Wolf-Rayet galaxy, NGC 3049, which has $\sim 500 \pm 240$ WR stars, to be used as template. In order to find out whether such a feature would be detected in a typical spectrum of our sample, we have extracted the WR bump from the spectra of NGC 3049 and superimposed it on one of the galaxies' spectrum. Taking into account the equivalent width, and the FWHM of the WR bump, we concluded that a typical spectrum of our sample has sufficient $\mathrm{S} / \mathrm{N}$ to detect a WR bump equivalent to at least $200 \mathrm{WR}$ stars. The fact that no WR feature is detected will not imply necessarily that there are no WR stars in the galaxy. It is possible that the WR feature is not strong enough to be detected.

Recently, we have started searching for features such as $\mathrm{H} \gamma$ which is a very prominent absorption line in A stars and should be easily detected given the $\mathrm{S} / \mathrm{N}$ of our spectra. However, we noticed that most of the $\mathrm{H} \gamma$ absorption lines are contaminated by emission which indicates the presence of $\mathrm{O}$ stars. We can also easily detect high order lines of the Balmer series in absorption. The spectra of A stars are rich in these lines.

Our next step is to search for He I lines, in particular, for He I $\lambda 4471$. This line seen in absorption is a strong evidence for the presence of very hot, massive, and young (O- and early B-type) stars in these galaxies.

\section{References}

Maia, M.A.G., Pastoriza, M.G., Bica, E., Dottori, H. 1994, ApJS 93, 425

Pastoriza, M.G., Bica, E., Maia, M.A.G., Bonatto, Ch., Dottori, H. 1994, ApJ 432, 128

Schaerer, D., Contini, T., Pindao, M. 1999, A\&AS 136, 35 